\title{
The correct author citation for taxa in Strumaria and changes to subgenera in Strumaria and Hessea (Amaryllidaceae: Amaryllideae), with a synopsis of the actinomorphic- flowered genera of subtribe Strumariinae
}

\section{Authors}

'D.A. Snijman (1)

1,2John C. Manning (D)

\section{Affiliations}

${ }^{1}$ Compton Herbarium, South African National Biodiversity Institute, Private Bag X7, Claremont 7735, South Africa.

${ }^{2}$ Research Centre for Plant Growth and Development, School of Life Sciences, University of KwaZuluNatal, Pietermaritzburg, Private Bag X01, Scottsville 3209, South Africa.

\section{Corresponding Author \\ J.C. Manning, \\ e-mail: J.Manning@sanbi.org.za}

\section{Dates}

Submitted: 25 August 2020

Accepted: 17 August 2021

Published: 10 September 2021

\section{How to cite this article:}

Snijman, D.A. \& Manning, J.C., 2021, 'The correct author citation for taxa in Strumaria and changes to subgenera in Strumaria and Hessea (Amaryllidaceae: Amaryllidaceae), with a synopsis of the actinomorphic-flowered genera of subtribe Strumaria', Bothalia 51(2), a9. http://dx.doi. org/10.38201/btha.abc.v51.i2.9

Copyright: (C) 2021. The Authors

Licensee: SANBI. This work is licensed under the Creative Commons Attribution 4.0 International License.

\begin{abstract}
Aspects of the nomenclature and classification of the subtribe Strumariinae are corrected and emended as follows: Hessea subgenus Myophila (Snijman) Snijman and Strumaria subgenus Carpolyza (Salisb.) Snijman are described, and Strumaria section Gemmaria (Salisb.) Snijman is validated; the correct author citations for several names in Strumaria that were invalidly published by Jacquin are established; and a complete infrageneric synopsis for the actinomorphic-flowered taxa of subtribe Strumariinae is provided.
\end{abstract}

Keywords: Africa; classification; Jacquin; new taxa; nomenclature; taxonomy; Willdenow.

\section{Introduction}

The predominantly African tribe Amaryllideae (Amaryllidaceae) includes four subtribes, of which Strumariinae Traub ex D.Müll.-Doblies \& U.Müll.-Doblies (Müller-Doblies \& Müller-Doblies 1985) are endemic to southern Africa (Meerow \& Snijman 2001). The complexity of relationships among the actinomorphic-flowered genera of Strumariinae is reflected in the differing taxonomic treatments of Carpolyza Salisb., Hessea Herb., Namaquanula D.Müll.-Doblies \& U.Müll.-Doblies and Strumaria Jacq. ex Willd. sensu lat. by Müller-Doblies and Müller-Doblies (1985) and Snijman (1994), based largely on these authors' interpretation of the group's floral morphology. Arising from the more recent molecular phylogenetic studies of Weichhardt-Kulessa et al. (2000) and Meerow and Snijman $(2001,2006)$, further taxonomic changes were implemented in the group. The monotypic Carpolyza was subsumed under Strumaria (Meerow \& Snijman 2001) and the genus Namaquanula was reinstated from its former rank as a subgenus in Hessea (Snijman 2005).

These changes necessitated a revision of the subgeneric classification of Hessea and Strumaria and the necessary adjustments are completed here. We recognise a new subgenus to accommodate Carpolyza spiralis (L'Hérit.) Salisb. within Strumaria and we validate the new section Gemmaria (Salisb.) Snijman within subgenus Gemmaria of Strumaria. We also formalise the change in rank of section Myophila Snijman to subgenus within Hessea. Further, we provide a synopsis of the current infrageneric classification for the actinomorphic-flowered Strumariinae, incorporating the taxonomic changes made since 1985 by Duncan and Voigt (2020), Weichhardt-Kulessa et al. (2000), Meerow and Snijman 
(2001), Müller-Doblies and Müller-Doblies (1992), and Snijman (1999, 2005).

We also correct the author citations of some species in Strumaria. The name Strumaria was first published by Jacquin (1795) in his Icones plantarum rariorum for five species of Amaryllideae from southern Africa's winterrainfall region. Although the individual species were described, no separate generic description or diagnosis was included, either then or later in his Collectaneorum supplementum (Jacquin 1797). This lack of a diagnosis for his new genus, which included more than a single species at the time, renders the generic name invalid (Turland et al., 2018: Art. 38.1, 38.5), and this in turn renders the names of the five species published by Jacquin under that genus also invalid (Turland et al., 2018: Art. 35.1). These names were all validated by Willdenow (1799) in the Species plantatum, fortunately without any change in their priority.

\section{Nomenclature}

\section{Corrections to author names}

Accepted names are in bold and synonyms in italics.

Strumaria Jacq. ex Willd., Species plantarum 2: 31 (1799). Lectotype: Strumaria truncata Jacq. ex Willd., designated by Phillips, Genera of South Afrcan Flowering Plants, ed. 2: 201 (1951).

Strumaria angustifolia Jacq. ex Willd., Species plantarum 2: 32 (1799). [Strumaria angustifolia Jacq.: 13 (1795), Jacq.: 48 (1797), invalid name, without generic description]. Type: illustration in Jacq.: t. 359 (1795).

Note: This taxon is considered to be conspecific with S. truncata Jacq. ex Willd. (Snijman 1994).

Strumaria filifolia Jacq. ex Willd., Species plantarum 2: 32 (1799), nom. illeg. superfl. pro Leucojum strumosum Sol. ex Aiton (1789). [Strumaria filifolia Jacq.: 14 (1795), invalid name, without generic description]. Type: as for Leucojum strumosum Sol. ex Aiton.

Note: Although Snijman (1994) considered this to be a legitimate name and typified it against the illustration in Icones plantarum rariorum 2: t. 361 (Jacquin, 1795), Jacquin (1795) clearly stated that he was coining it as a preferred replacement name for Leucojum strumosum ["melius quam Leucojum strumosum"] with a direct reference to Aiton (1789), and it is therefore correctly treated as an illegtimate superfluous name for that taxon, with the same type. It is considered to be conspecific with S. tenella (L.f.) Snijman (Snijman 1994).

Strumaria linguifolia Jacq. ex Willd., Species plantarum 2: 31 (1799). [Strumaria linguifolia [as 'linguaefolia']
Jacq.: 13 (1795), Jacq.: 45 (1797), invalid name, without generic description]. Type: illustration in Jacq.: t. 356 (1795).

Note: This taxon is considered to be conspecific with S. truncata Jacq. ex Willd. (Snijman 1994).

Strumaria rubella Jacq. ex Willd., Species plantarum 2: 31 (1799). [Strumaria rubella Jacq.: 13 (1795); Jacq.: 46 (1797), invalid name, without generic description]. Type: illustration in Jacq.: t. 358 (1795).

Note: This taxon is considered to be conspecific with S. truncata Jacq. ex Willd. (Snijman 1994).

Strumaria truncata Jacq. ex Willd., Species plantarum 2: 31 (1799). [Strumaria truncata Jacq.: 13 (1795), Jacq.: 47 (1797), invalid name, without generic description]. Type: illustration in Jacq.: t. 357 (1795).

Strumaria undulata Jacq. ex Willd., Species plantarum 2: 32 (1799). [Strumaria undulata Jacq.: 14 (1795), Jacq.: 50 (1797), invalid name, without generic description. Type: illustration in Jacq.: t. 360 (1795).

Note: The identity of this taxon is uncertain (Snijman 1994).

\section{New subgenera and sections}

Strumaria Jacq. ex Willd., Species plantarum 2: 31 (1799).

Subgenus Carpolyza (Salisb.) Snijman, stat. nov. Carpolyza Salisb., Paradisus Londinensis 1: 63 (1807). Type: Carpolyza spiralis (L'Hérit.) Salisb. = Strumaria spiralis (L'Hérit.) Aiton

Section Gemmaria (Salisb.) Snijman, sect. nov. [Strumaria subg. Gemmaria sect. Gemmaria, invalid name without author, Snijman: 106 (1994)]. Gemmaria Salisb., The Genera of Plants: 127 (1866). Type: as for Gemmaria Salisb.

Hessea Herb., Amaryllidaceae: 289 (1837).

Subgenus Myophila (Snijman) Snijman, stat. nov. Hessea subgenus Namaquanula section Myophila Snijman in Contributions from the Bolus Herbarium 16: 76 (1994). Type: Hessea mathewsii W.F.Barker

\section{Synopsis of infrageneric taxa in Strumaria, Hessea and Namaquanula}

Strumaria Jacq. ex Willd., Species plantarum 2: 31 (1799). Lectotype: Strumaria truncata Jacq. ex Willd., designated by Phillips, Genera of South African Flowering Plants, ed. 2: 201 (1951).

\section{Subgenus Strumaria}

Bulb tunics parchment-like, whitish. Cataphyll present, sometimes exserted. Foliage leaves (2)3 or 
4(6), erect, spreading laterally in a fan, lorate, glabrous, rarely sticky. Scape persisting beyond seed release. Flowers funnel-shaped, rarely hypocrateriform or campanulate, pedicels more-or-less as long as perianth, tepals free, filaments usually connate into a tube with outer whorl adnate to style, anthers dorsifixed, style 3-angled or -winged towards base, rarely uniformly swollen. Chromosome base number $x=10$.

8 spp.: S. barbarae Oberm. [as 'barbariae' Oberm.], S. bidentata Schinz, S. hardyana D.Müll.-Doblies \& U.Müll.-Doblies, S.luteoloba Snijman, S. phonolithica Dinter, S. prolifera Snijman, S. speciosa Snijman, S. truncata Jacq. ex Willd.

Subgenus Carpolyza (Salisb.) Snijman [validated above]. Carpolyza Salisb.: 63 (1807). Type: Carpolyza spiralis (L'Hérit.) Salisb.

Bulb tunics thinly fibrous, whitish. Cataphyll absent. Foliage leaves 4 to 6 , spreading, filiform, glabrous. Scape \pm spirally twisted proximally, persisting beyond seed release. Flowers funnel-shaped, pedicels shorter to much longer than perianth, tepals connate into a short tube basally, filaments decurrent on perianth tube with inner whorl shortly adnate to style, anthers subcentrifixed, style somewhat 3angled. Chromosome base number $x=10$.

1 sp.: S. spiralis (L'Hérit.) Aiton

Subgenus Tedingea (D.Müll.-Doblies \& U.Müll.-Doblies) Snijman in Contributions from the Bolus Herbarium 16: 86 (1994). Tedingea D.Müll.-Doblies \& U.MülI.-Doblies: 45 (1985). Type: S. tenella (L.f.) Snijman Bulb tunics softly fibrous, whitish. Cataphyll present or absent. Foliage leaves 2 to 6, spreading, filiform, glabrous. Scape often proximally flexed or spirally twisted, usually persisting during seed release. Flowers stellate, rarely somewhat campanulate, pedicels much exceeding perianth length, tepals free, filaments separate, both whorls adnate to swollen style base, anthers dorsifixed. Chromosome base number $x=10$.

2 spp.: S. pygmaea Snijman, S. tenella (L.f.) Snijman subsp. tenella, S. tenella subsp. orientalis Snijman

Subgenus Gemmaria (Salisb.) Snijman in Contributions from the Bolus Herbarium 16: 105 (1994). Gemmaria Salisb.: 127 (1866). Type: Gemmaria gemmata (Ker Gawl.) Salisb. ex D.Müll.-Doblies \& U.Müll.-Doblies $=S$. gemmata Ker-Gawl.

Bulb tunics parchment-like, whitish or yellowish. Cataphyll present or rarely absent. Foliage leaves 2(3), spreading to prostrate, lorate to elliptic, plane, more-or-less pubescent or pustulate, at least in juveniles. Scape mostly detaching basally at seed set. Flowers stellate to funnel-shaped, pedicels shorter or longer than perianth, tepals free, filaments separate, both whorls adnate to swollen style base, anthers subcentrifixed. Chromosome base number $x=10$.

Note: The phylogenetic study of Meerow and Snijman (2001), using nrDNA ITS sequences and morphology, shows weak support for the currently recognised sections of subgenus Gemmaria. The results, however, are based on less than a third of the species of the subgenus. Accordingly, until a more complete analysis of the clade becomes available, we retain the sections recognised in Snijman (1994).

Section Gemmaria (Salisb.) Snijman [validated above]. Gemmaria Salisb.: 127 (1866). Type: as for subgenus. Bulb tunics whitish or yellowish. Cataphyll present. Foliage leaves 2(3). Flowers stellate, pedicels at least twice as long as perianth, filaments attached to broad style base, style distinctly widest at base.

11 spp.: S. argillicola G.D.Duncan, S. chaplinii (W.F.Barker) Snijman, S. discifera Marloth ex Snijman subsp. discifera, $S$. discifera subsp. bulbifera Snijman, S. gemmata Ker Gawl., S. karooica (W.F.Barker) Snijman, S. karoopoortensis (D.Müll.Doblies \& U.Müll.-Doblies) Snijman, S. leipoldtii (L.Bolus) Snijman, S. massoniella (D.Müll.-Doblies \& U.Müll.-Doblies) Snijman, S. merxmuelleriana (D.Müll.-Doblies \& U.Müll.-Doblies) Snijman, S. unguiculata (W.F.Barker) Snijman, S. villosa Snijman

Section Bokkeveldia (D.Müll.-Doblies \& U.Müll.Doblies) Snijman in Contributions from the Bolus Herbarium 16: 131 (1994). Bokkeveldia D.Müll.Doblies \& U.Müll.-Doblies: 27 (1985). Type: Bokkeveldia watermeyeri (L.Bolus) D.Müll.-Doblies \& U.Müll.-Doblies $=$ S. watermeyeri L.Bolus

Bulb tunics whitish or yellowish. Cataphyll present. Foliage leaves 2(3). Flowers more-or-less funnelshaped, pedicels slightly shorter or longer than perianth, filaments adnate to style for 2.5 to $4.0 \mathrm{~mm}$ above base, style more-or-less evenly thickened in proximal half.

5 spp.: S. aestivalis Snijman, S. perryae Snijman, S. pubescens W.F.Barker, S. salteri W.F.Barker, S. watermeyeri L.Bolus subsp. watermeyeri, S. watermeyeri subsp. botterkloofensis (D.Müll.-Doblies \& U.Müll.-Doblies) Snijman

Section Cryptomeria Snijman in Contributions from the Bolus Herb. 16: 105 (1994). Type: S. picta W.F.Barker

Bulb tunics whitish. Cataphyll absent or rarely present. Foliage leaves usually more than 3, only 2 exserted above ground, shortly ciliate. Flowers weakly campanulate, pedicels somewhat longer than perianth, minutely pubescent, filaments thickened and ventrally ridged in proximal half, attached to style 
base, style narrowly ovoid, 6-grooved proximally, tapering distally.

1 sp.: S. picta W.F.Barker

Hessea Herb., Amaryllidaceae: 289 (1837). Type: Hessea stellaris (Jacq.) Herb.

\section{Subgenus Hessea}

Hessea subgenus Kamiesbergia (Snijman) Snijman: 71 (1994); Snijman: 109 (1999). Kamiesbergia Snijman: 125 (1991). Type: Kamiesbergia stenosiphon Snijman $=H$. stenosiphon (Snijman) D.Müll.Doblies \& U.Müll.-Doblies

Bulb tunics parchment-like. Cataphyll present. Foliage leaves 2, glabrous or rarely minutely pilose. Flowers stellate, somewhat funnel-shaped or rarely hypocrateriform, tepals often adnate to filaments, filaments connate proximally into a short to long tube, rarely inner and outer whorls dissimilar, smooth, anthers centrifixed, ovary dome flattened. Chromosome base number $x=11$.

11 spp.: $H$. breviflora Herb., $H$. cinnamomea (L'Hérit.) T.Durand \& Schinz, H. incana Snijman, H. monticola Snijman, H. pilosula D.Müll.-Doblies \& U.Müll.-Doblies, $H$. pusilla Snijman, $H$. speciosa Snijman, H. stellaris (Jacq.) Herb., H. stenosiphon (Snijman) D.Müll.-Doblies \& U.Müll.-Doblies, H. tenuipedicellata Snijman, $H$. undosa Snijman
Subgenus Myophila (Snijman) Snijman [validated above]. Hessea section Myophila Snijman: 76 (1994). Type: H. mathewsii W.F.Barker

Bulb tunics softly fibrous. Cataphyll present. Foliage leaves 2(3), glabrous. Flowers stellate, tepals free to base, filaments free or shortly connate basally, densely papillate adaxially and each bearing a curved blunt hook arching over central disc, anthers subcentrifixed, ovary dome raised into 3 trichome-covered green pulvini between style and inner whorl. Chromosome base number $x=11$.

2 spp.: H. mathewsii W.F.Barker, H. pulcherrima (D. Müll.-Doblies \& U.Müll.-Doblies) Snijman

Namaquanula D.Müll.-Doblies \& U.Müll.-Doblies in Botanische Jahrbücher 107: 20 (1985); Snijman: 155 (2005), emend. Hessea subgenus Namaquanula (D.Müll.-Doblies \& U.Müll.-Doblies) Snijman: 74 (1994), excluding section Myophila Snijman. Type: N. bruce-bayeri D.Müll.-Doblies \& U.Müll.-Doblies

Bulb tunics brittle, tan-coloured. Cataphyll absent. Foliage leaves (1)3 or 4, glabrous. Flowers stellate, tepals shortly connate or free, filaments proximally papillate on adaxial surface, anthers dorsifixed. Chromosome base number $x=11$.

2 spp.: N. bruce-bayeri D.Müll.-Doblies \& U.Müll.Doblies, N. bruynsii Snijman

\section{References}

Aiton, W., 1789, Hortus kewensis, vol. 1, George Nicol, London.

Duncan, G. \& Voigt, L., 2020, 'Strumaria argillicola', Curtis's Botanical Magazine 37(1): 12-24, https://doi.org/10.1111/ curt.12317.

Herbert,W., 1837, Amaryllidaceae, James Ridgway, London.

Jacquin, N.J., 1795, Icones plantarum rariorum, vol. 2, fasc. 16. Wappler, Vienna etc.

Jacquin, N.J., 1797, Collectaneorum supplemetum, Wappler, Vienna.

Meerow, A.W. \& Snijman, D.A., 2001, 'Phylogeny of Amaryllidaceae tribe Amaryllideae based on nrDNA ITS sequenes and morphology', American Journal of Botany 88(2): 2321-2330.

Meerow, A.W. \& Snijman, D.A., 2006, 'The never-ending story: multigene approaches to the phylogeny of Amaryllidaceae', Aliso 22: 355-366.

Müller-Doblies, D. \& Müller-Doblies, U., 1985, 'De Liliifloris notulae 2. De taxonomia subtribus Strumariinae (Amaryllidaceae)', Botanische Jahrbücher für Systematik 107: 17-47.

Müller-Doblies, D. \& Müller-Doblies, U., 1992, 'De Liliifloris notulae 4. Notes on Hessea (including Kamiesbergia: Amaryllidaceae of southern Africa)', Gleditschia 20: 1520.
Phillips, E.P., 1951, The genera of South African Flowering Plants, edn. 2, Memoirs of the Botanical Survey of South Africa 25.

Salisbury, R.A., 1807, The paradisus londinensis, vol. 1(2), W.Hooker, London.

Salisbury, R.A., 1866, The genera of plants. A fragment containing part of Liriogamae, John van Vöorst, London.

Snijman, D.A., 1994, 'Systematics of Hessea, Strumaria and Carpolyza (Amaryllideae: Amaryllidaceae)', Contributions from the Bolus Herbarium 16, University of Cape Town and National Botanical Institute, South Africa.

Snijman, D.A., 1999, 'A new species, notes on subgeneric taxa, and new synonyms in Hessea (Amaryllidaceae: Amaryllideae) from South Africa', Novon 9: 107-110.

Snijman, D.A., 2005, 'A new species of Namaquanula (Amaryllidaceae: Amaryllideae) from Namibia with notes on the genus', Bothalia 13(2): 153-156, https://doi.org/10.4102/ abc.v35i1.390.

Turland, N.J., Wiersma, J.H., Barrie, F.R., Grueter, W., Hawksworth, D.L., Herendeen, P.S., Knapp, S., Kusber, W.-H., Li, D.-Z., Marhold, K., May, T.W., Mcneil, J., Monro, A.M., Prado, J., Price, M.J. \& Smith, G.F. (eds), 2018. International Code of Nomenclature for algae, fungi, and plants (Shenzhen Code) adopted by the Nineteenth International Botanical Congress Shenzhen, China, July 2017. Regnum Vegetabile 159. Glashütten: Koeltz Botanical Books. 
Weichhardt-Kulessa, K., Börner, T., Schmitz, J., MüllerDoblies, D. \& Müller-Doblies, U., 2000, 'Controversial taxonomy of Strumariinae (Amaryllidaceae) investigated by nuclear rDNA (ITS) sequences. 1. Hessea, Namaquanula,
Kamiesbergia, and Dewinterella', Plant Systematics and Evolution 223: 1-13.

Willdenow, C.L., 1799, Species plantarum, edn. 4, vol. 2, Nauk, Berlin. 